\title{
Microbiology Susceptibility Reason Not Done
}

National Cancer Institute

\section{Source}

National Cancer Institute. Microbiology Susceptibility Reason Not Done. NCI Thesaurus.

Code C87937.

The explanation given as to why microbiology susceptibility was not assessed. 